\title{
Approximate method for calculating a thick- walled cylinder with rigidly clamped ends
}

\author{
Vladimir Andreev ${ }^{1, *}$ \\ ${ }^{1}$ Moscow State University of Civil Engineering, Yaroslavskoe shosse, 26, Moscow, 129337, Russia
}

\begin{abstract}
Numerous papers dealing with the calculations of cylindrical bodies [ $1-8$ and others] have shown that analytic and numerical-analytical solutions in both homogeneous and inhomogeneous thick-walled shells can be obtained quite simply, using expansions in Fourier series on trigonometric functions, if the ends are hinged movable (sliding support). It is much more difficult to solve the problem of calculating shells with builtin ends.
\end{abstract}

\section{Introduction}

As was shown in [8], the natural restriction of the method of separation of variables in the calculation of radially inhomogeneous cylinders is the impossibility of satisfying arbitrary boundary conditions at the ends. This paper considers an approximate method for solving the axisymmetric problem for a thick-walled cylinder with rigid fixed ends. The problem is solved in displacements.

\section{State of the problem}

In solving the axisymmetric problem will be sought displacements in the form:

$$
\begin{array}{rl}
u(r, \theta, z) & =\sum_{m=0}^{\infty} \sum_{n=0}^{\infty} u_{m n}(r) \cdot \cos m \theta \cdot \cos k_{n} z \\
v(r, \theta, z)=\sum_{m=1}^{\infty} \sum_{n=0}^{\infty} v_{m n}(r) \cdot \sin m \theta \cdot \cos k_{n} z & w(r, \theta, z)=\sum_{m=0}^{\infty} \sum_{n=1}^{\infty} w_{m n}(r) \cdot \cos m \theta \cdot \sin k_{n} z
\end{array}
$$

From (1), the boundary conditions automatically follow

\footnotetext{
*Corresponding author: asv@mgsu.ru
} 


$$
z=0, H ; \quad w=\tau_{r z}=\tau_{\theta z}=0
$$

which correspond to the hinged (sliding) support ( $H$ - cylinder height).

Below we consider an approximate calculation method cylinder with rigid fixing ends. In this case, the boundary conditions at the ends are written in the form:

$$
z=0, H ; \quad u=w=0 .
$$

Fig. 1 shows half the cross-section of a cylindrical shell for two ways of fixing the ends.
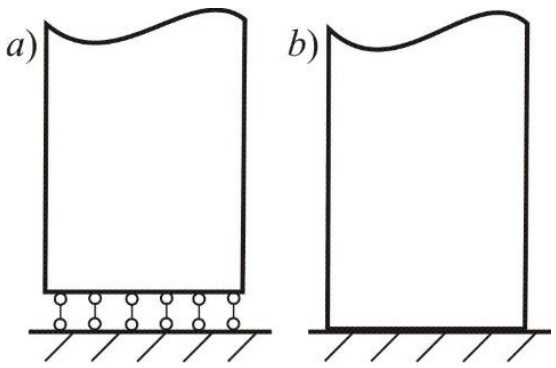

Fig 1. Thick-walled cylinder, $a$ - hinged movable support, $b$ - built-in (rigid fixing) end.

\section{Approximate method for calculating}

The proposed method is based on the algorithm for calculating cylinders for the action of surface loads, which use an expanded area, compensating loads and the collocation method. We consider the problem when the external loads are symmetric with respect to the plane $z_{1}=H / 2$ (Fig. 2). Let us increase the height of the cylinder in both directions by an amount $\delta$ and introduce the coordinate $z=z_{1}+\delta$ We apply to the lateral surfaces of the cylinder in the interval $[0, \delta]$ and $[H+\delta, H+2 \delta]$ such compensating loads $p_{a}^{c}, p_{b}{ }^{c}, q_{a}{ }^{c}, q_{b}{ }^{c}$, that at the given points $r=r_{1}$ and $r=r_{2}$ at $z=\delta$ and $z=\delta+H$ (Fig. 3) the following conditions are satisfied:

$$
r=r_{1}, r_{2} ; \quad u=w=0 .
$$

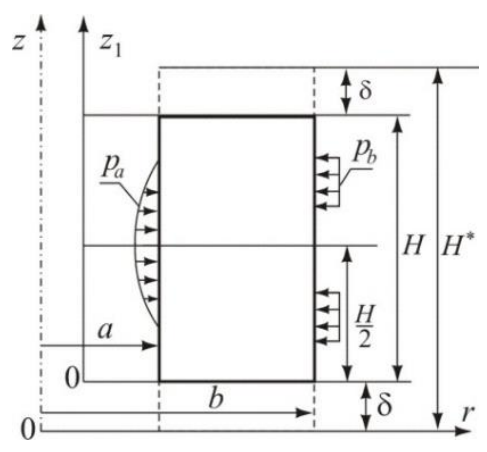

Fig. 2. Calculation scheme for a cylinder with an expanded area.

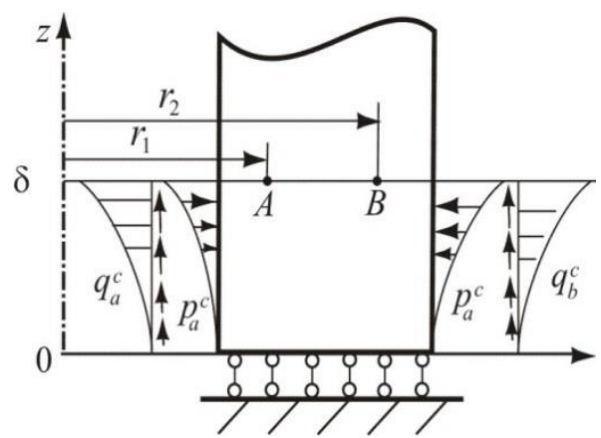

Fig. 3. Compensating loads in expanded area.

If equalities (4) are considered as conditions of real fixation, then it should be added that at the other points of the layers $z=\delta$ and $z=\delta+H$ the displacements can be either positive or negative. In this case, it is necessary to accept the hypothesis of an ideal contact between the ends of the cylinder and the support surfaces, as is done in most contact 
problems, for example, in the calculation of beams on an elastic foundation. Let us consider the nature of the compensating loads. If we imagine that these loads are the reaction of a semi-infinite array to the walls of a cylinder embedded in it, then as the distance from the layer $z=\delta$ to the layer $z=0$, the tangential and normal loads should tend to zero. For a description of such loads can be approximately selected functions of the form:

$$
f=\left\{\begin{array}{lc}
f_{0}\left(\frac{z}{\delta}\right)^{\gamma} & 0 \leq z \leq \delta ; \\
0 & \delta \leq z \leq \frac{H}{2}+\delta .
\end{array}\right.
$$

Such functions well approximate normal loads and worse - tangents, which near the layer $z=\delta$ have a peak character and when $z=\delta$ they are equal to zero. Nevertheless, if we bear in mind that when the compensating loads are decomposed into Fourier series, the peak nature of the loads $q_{a}^{c}$ and $q_{b}^{c}$ will also correspond quite accurately to their representations in the form of a series (Fig. 4).

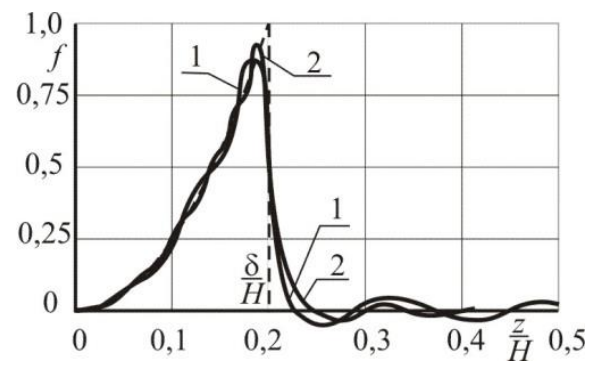

Fig. 4. Representation of function $f$ by the Fourier series : - - - formula (5), _ Fourier series $(1-N=24 ; 2-N=48)$.

The essence of this method is as follows. Representing compensating loads $p_{a}^{c}, p_{b}{ }^{c}$, $q_{a}^{c}, q_{b}^{c}$, in the form (5) and varying the parameters $\delta$ and $\gamma$, it is necessary to achieve the conditions (4) at the points $A$ and $B$ (see Fig. 3). It should be added that when using the collocation method, large deficiency of conditions (4) are possible at the remaining points of the interval $(a, b)$ on the layer $z=\delta$. Therefore, the choice of the above parameters of the functions (5) must also be accompanied by an analysis of the indicated deficiency and its minimization. In this case, it is still possible to select the radii $r_{1}$ and $r_{2}$, determining the position of the points $A$ and $B$.

The following sequence of solution of the problem is proposed. At the first stage, using the numerical-analytical method presented in $(7,8)$, the problem of the effect of external force $\left(p_{a}, p_{b}, q_{a}, q_{b}\right)$ and temperature loads on a cylinder of length $H+2 \delta$ is solved. At the same time presence of hinged (sliding) supports at the ends $z=0$ and $z=H+2 \delta$, give the possibility of using the method. As a result of the solution, displacements $u_{P}, w_{P}$ at points $A$ and $B$ are determined.

The index $p$ denotes the displacements from external (active) loads. The condition of symmetry with respect to the plane $z=H / 2+\delta$ allows us to restrict ourselves to the consideration of displacements on the layer $z=\delta$. 
Further problems are solved successively to the action of compensating loads $p_{a}^{c}, p_{b}^{c}, q_{a}^{c}$ and $q_{b}^{c}$, as defined in (5). The highest values of these loads are assumed to be equal to unity (unit loads). Defining the displacements from unit loads at points $A$ and $B$, one can construct a system of algebraic equations corresponding to conditions (4):

$$
U^{c} \cdot K+U_{P}=0
$$

where $U^{c}$ is the displacements matrix of unit compensating loads of $4 \times 4$ :

$$
U^{c}=\left(\begin{array}{cccc}
u_{1 A}^{c} & u_{2 A}^{c} & u_{3 A}^{c} & u_{4 A}^{c} \\
w_{1 A}^{c} & w_{2 A}^{c} & w_{3 A}^{c} & w_{4 A}^{c} \\
u_{1 B}^{c} & u_{2 B}^{c} & u_{3 B}^{c} & u_{4 B}^{c} \\
w_{1 e}^{c} & w_{2 e}^{c} & w_{3 e}^{c} & w_{4 e}^{c}
\end{array}\right)
$$

where the subscripts $1 \div 4$ denote respectively the displacements from the loads $p_{a}^{c}, p_{b}^{c}, q_{a}^{c}$ and $q_{b}^{c} ; \quad K=\left\{K_{1}, K_{2}, K_{3}, K_{4}\right\} \quad$ is the vector of unknown coefficients; $U_{P}=$ $\left\{u_{P A}, w_{P A}, u_{P B}, w_{P B}\right\}$ is the displacements vector from the active loads, including the displacements $u_{P}, w_{P}$ at points $A$ and $B$.

The coefficients $K_{1} \div K_{4}$, found as a result of solving the system of equations (6), determine the actual values of the compensating loads at the level $z=\delta$. In other words, the compensating loads needed for the fulfillment of the conditions (4) in the section $0 \leq z \leq \delta$ will have the form:

$$
p_{a}^{c}=K_{1}\left(\frac{z}{\delta}\right)^{\gamma} ; \ldots q_{b}^{c}=K_{4}\left(\frac{z}{\delta}\right)^{\gamma}
$$

Summarizing the solutions of five problems (one from external and four from compensating loads), we obtain the final solution of the problem that satisfies the boundary conditions.

\section{Analysis of the influence of various factors on the calculation results}

As noted above, the proposed method has three arbitrary factors:

- length of the fixed edge $\delta$;

- degree of compensation load functions $\gamma$;

- choice of collocation points $A$ and $B$.

Investigation of the influence of these factors on the stress-strain state of the cylinder and the determination of optimal calculation parameters was carried out in solving the problem for a homogeneous cylinder. We also considered the question of choosing the number of terms of the Fourier series $N$ in the decomposition of loads and the number of steps $M$ into which the integration interval is divided in the numerical solution of systems of ordinary differential equations. 
On the example of the solution of the problem for a homogeneous cylinder with hinged (sliding) ends under the action of a constant external pressure $p_{b}=p$, let us analyze the influence of the number $M$ on the calculation results. Obviously, in this case there is a problem of a flat deformed state, for which there is an analytic solution [4]. In particular, the axial stresses in this problem are equal to:

$$
\sigma_{z}=-2 v \frac{p b^{2}}{b^{2}-a^{2}} .
$$

When choosing the number $M$, a comparison is made of the stress values. At $M=30$, the difference between numerical and analytical calculations did not exceed 1\%. An analysis of the influence of the number of terms of the Fourier series $N$ on the results of calculations showed that at $N=24$ and $N=48$, the differences in the compensating loads (see Fig. 3 ) at individual points reach $6 \%$. At the same time, when calculating stresses and displacements, the difference does not exceed 3\%, which is due to the Saint Venant principle. The results presented below were obtained at $N=24$.

We now turn to an analysis of the effect of the parameters of the method of compensating loads on the value of the deficiency of the boundary conditions (4) on the interval $(a, b)$ with the exception of the collocation points $A$ and $B$, where these conditions are satisfied exactly. Let us consider the problem of calculating homogeneous cylinder loaded with an external constant pressure $p_{b}=p$ with rigidly clamped (built-in) ends. Note that when considering an elongated cylinder whose height is equal $H^{*}=H+2 \delta$, the pressure $p$ is applied to the section $(\delta, H+\delta)$. The corresponding formulas for the coefficients of the expansion of this load in a Fourier series have the form: $p_{b, 0}=p H / H^{*}$;

$$
p_{b, n}=\frac{2 p}{n \pi}\left[\sin k_{n}\left(H^{*}-\delta\right)-\sin k_{n} \delta\right], \text { where } k_{n}=n \pi / H^{*}
$$

Calculations were made for the cases when the compensating loads are represented as a linear function, quadratic and cubic parabolas, to which correspond to the values of the parameter entering in (5) equal to 1,2 and 3.

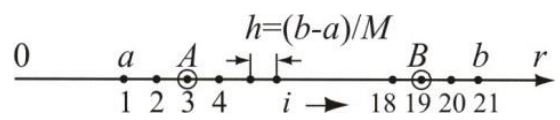

Fig. 5. Arrangement of collocation points

In addition, the depth of the "fixed edge" $\delta$ within the range of $0,05 H \div$ $0,5 H$ varied in the calculations., as well as the location of the collocation points, which were selected along the borders of the sections to which the integration interval $(a, b)$ was divided. For $M=20$, the number of borders of the sections is 21 . Fig. 5 shows, as an example, the location of the points $A$ and $B$ at points with the numbers $i=3$ and $i=19$.

An analysis of the discrepancy between the boundary conditions on the layer $z=\delta$ was carried out by comparing the largest displacements of the compensating loads $u^{c}$ and $w^{c}$ on this layer with the largest displacements $u^{p}$ in the middle section of the cylinder from the pressure $p$ (the displacements $w^{p}$ are negligibly small in comparison with $u^{p}$ ). A similar analysis was also conducted with respect to the choice of the optimal value and the depth of the "fixed edge" $\delta$. It is established that the smallest discrepancy between the boundary conditions 
is reached at $\gamma=2$. As regards the influence $\delta$ on the displacements of the points of the layer $z=\delta$, the largest displacements in this layer at $\delta>0,2 H$ remain practically constant (Fig 6). At the same time, within the range of $0.05 H \div 0.5 H$, the stresses $\sigma_{z}$ change significantly. For $\delta \cong 0,5 H$ the function $\bar{\sigma}_{z}=\sigma_{z} / p$ asymptotically approaches some limiting values.

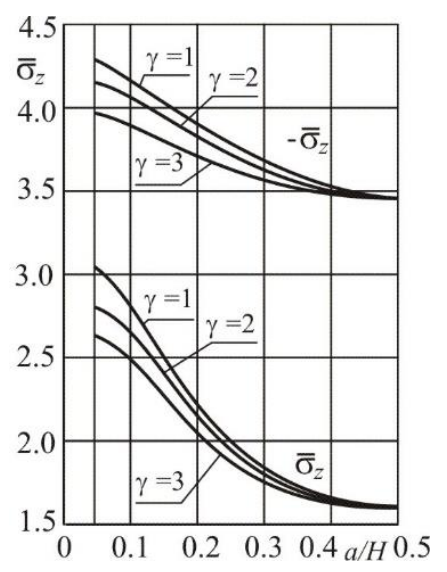

Fig. 6. Dependences stresses $\bar{\sigma}_{z}$ from $\delta$ at different values $\gamma$. Collocation points: 1 and 21 .

Fig. 7 shows the graphs of the variation stresses $\bar{\sigma}_{z}$ along the axis $z$ on the inner and outer surfaces of the cylinder, calculated with the following initial data and calculation parameters: $H=2 \mathrm{~m} ; a=0.5 \mathrm{~m} ; b=1 \mathrm{~m} ; E=2.10^{4} \mathrm{MPa} ; v=0.2 ; v=0.2 ; N=20 ; M=24$; $\gamma=2 ; \delta=1 \mathrm{~m}$; the collocation points are 1 and 21 . In the same figure, the values of the stresses in the cylinder with the hinged (sliding) support of the ends are shown in dotted lines. In the latter case, as noted above, the problem corresponds to a flat deformed state, and the stresses $\sigma_{z}$ are constant over the cross section (see (8)), and they are identical in all cross sections.

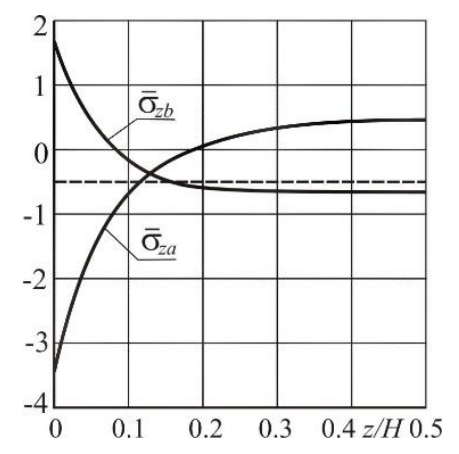

Fig. 7. Changing stresses $\bar{\sigma}_{z}$ along the axis $z$ in the cylinder with: - - - - sliding support of the ends; - rigidly clamping ends.

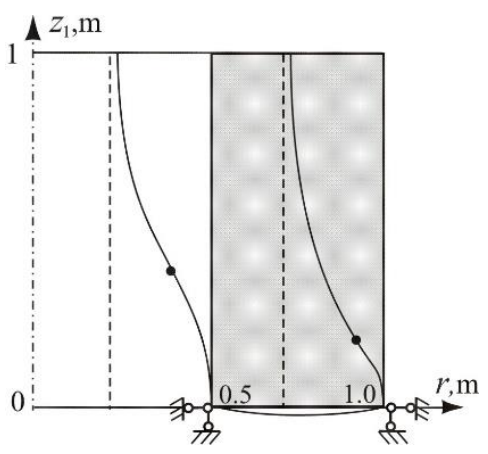

Fig. 8. Deformed state of the cylinder, - - - - sliding support, _ rigidly clamping ends, - - the inflection points.

In order to fully appreciate the difference in the results obtained in solving two problems, we consider the deformed states of cylinders with hinged support of the ends and with rigidly 
clamped ends (Fig. 8). At the collocation points $(r=a, b)$ in Fig. 8 fixed supports are installed. Please pay attention to the following features. The deformation of the cylinder wall in the presence of a rigid clamping of the ends is qualitatively similar to the bending of a beam with clamped ends. Near the support, tensile stresses are observed from the outside, and compression is observed inside. The inflection points indicate a change in the sign of the deformations $\varepsilon_{z}$. Both recent facts agree with the stress plots in Fig. 6. Negative displacements $w$ at the level $z_{1}=0$ are caused by the expansion of the cylinder in the axial direction due to the Poisson ratio.

\section{Conclusions}

Comparison of displacements and stresses in a cylinder with sliding support along the ends and in cylinder with rigidly clamped ends shows a significant influence of the type of boundary conditions on the stress-strain state of the cylinders. In sufficiently tall cylinders, this effect will act as a marginal effect, and in short cylinders and cylinders of medium height, the influence of the boundary conditions will affect the entire height [9].

\section{This work was financially supported by the Ministry of Russian Education (7.6163.2017/BУ)}

\section{References}

1. Filon L.N.G., Phil. Trans. of the Royal Society of London, Ser. A.. V. 198. 4, 147 (1902)

2. Prokopov V.K., PMM. V. XIII 2, 135 (1949)

3 Volkov A.N., Calculation of thick-walled hollow cylinders (RUDN, Moscow, 1972)

4. Timoshenko S.P., Gudier Dj., Theory of elasticity. (Nauka, Moscow, 1979)

5. Pankratova N.D., Thermal stress in structural elements. 20 Kiev, 63(1980)

6. Andreev V.I., Proc. of high schools. Ser. build. and architect. 8, 27 (1985)

7. Andreev V.I., Constr. mech. \& calc. str. 2, 8 (1989)

8. Andreev V.I., Some problems and methods of mechanics of inhomogeneous bodies, (ASV, Moscow, 2002)

9. Andreev V.I., Applied Mechanics and Materials. 166-169, 354 (2012) 